\title{
Application of Improved Fuzzy-Smith Controller in the Control System of Cement Rotary Kiln
}

\author{
Haiyan Zhang \\ College of Information and Control Engineering, Weifang University, Weifang, China \\ wfxx_zhy@163.com
}

Keywords: Fuzzy-smith control; Rotary cement kiln; Pure lag; Robustness

\begin{abstract}
Rotary cement kiln is a large time delay and inertia component. It is typical problems in industrial process control, so when applying advanced control methods to systems. This paper designs an improved Fuzzy-Smith controller. It combines Fuzzy with improved Smith predictor control method. Smith predictor algorithm compensates for the time delay and Fuzzy control is used to enhance the robustness of the system. The simulation shows that the improved Fuzzy-Smith controller owns stronger robustness and much better control quality. And it is an appropriate and simple control method for the system with pure lag.
\end{abstract}

\section{Introduction}

In solving the large lag controlled system of cement rotary kiln, Smith predictive control is often used as a control method. The greatest advantage of Smith predictive control is to move the time-delay link to the closed loop. The biggest drawback is that it relies too much on the precise mathematical model of the controlled object $[1,2]$. The fuzzy control system has the characteristics of not requiring accurate model and strong robustness, but the fuzzy control cannot overcome the influence of large lag [3,4]. Now Fuzzy-Smith control has been widely used in this large lag and large inertia link, and has achieved good control effect [5-8]. In the Fuzzy-Smith control scheme, although the fuzzy controller is not sensitive to the change of the model parameters, the fuzzy controller is also powerless when the model of Smith predictor is quite different from the object model. The conventional PID regulator is used to control such objects, but the stability time is longer, and it cannot meet the requirements well [9]. In this paper, an improved Fuzzy-Smith intelligent controller is studied, which combines fuzzy control with improved Smith predictive control scheme, thus improving the stability and robustness of the control system.

\section{Smith Predictive Control}

Smith predictive control method with pure lag object is simplified as with pure lag object control provided in theory, the basic idea is in accordance with the dynamic characteristics of the process to build a model into a feedback control system, which was delayed by the delay time for the amount charged ahead of $\tau$ reflects to the controller. Let the controller work in advance, which can significantly reduce the overshoot and accelerate the adjustment process. The block diagram of its control system is shown in Fig. 1, in which $\mathrm{W}$ (s) represents the transfer function of the controller, $G_{p}(s) e^{-\tau s}$ is the transfer function of the controlled object, $G_{p}(s)$ is the transfer function with no pure lag part in the controlled object, $e^{-\tau s}$ is the transfer function of the pure lag part of the controlled object [10]. 


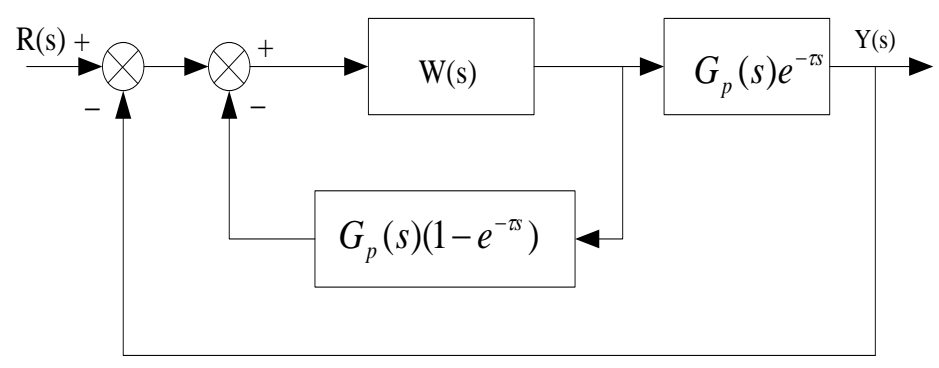

Figure 1. Principle diagram of Smith predict control

The basic principle of Smith predictive control is $G_{p}(s) e^{-\tau s}$ parallel one compensation link $G_{p}(s)\left(1-e^{-\tau s}\right)$, which is used to compensate the pure lag part in the controlled object. The closed loop transfer function of the system after compensation is Eq. 1.

$$
\Phi(s)=\frac{W(s) G_{p}(s)}{1+W(s) G_{p}(s)} e^{-\tau s}
$$

The formula (1) shows that the closed loop characteristic equation of the compensated system does not contain the pure lag part $e^{-\tau s}$, so the effect of the pure lag part on the control system is eliminated.

\section{Design of Fuzzy-Smith Intelligent Controller}

Smith predictive control delay in solving this cement rotary kiln controlled system, is considered to be the most effective way, but the traditional Smith controller in W(s) is a PID controller. The PID control is designed based on the accurate model of controlled object. Therefore, for cement rotary kiln, it is difficult to achieve satisfactory control effect due to the lack of precise models and time-varying parameters and process control. Large overshoot and severe oscillation often occur, the reason is that the amount transferred by the system can not reflect the disturbance, adjust the controller need through the pure time lag before affected by adjustment, when $\tau$ is large, there will be the phenomenon. For fuzzy control, although it doesn't need to know the exact mathematical model of the controlled object and has good robustness, it is not very ideal for large pure time-delay process. With the increase of pure time lag, the properties of the fuzzy controllers will deteriorate, appears overshoot and oscillation.

In view of the above characteristics, fuzzy controller and Smith predictive controller are combined to compensate for the shortcomings of Smith controller and fuzzy controller, so that it is satisfied with the production process of cement rotary kiln with large delay characteristics. The Smith estimation method is used to estimate the output of the system, and W (s) in Figure 1 is the transfer function of the fuzzy controller. Prediction of pure lag compensation system controller with Smith, aiming at the inaccuracy of the controlled object model and the prediction model, the design of fuzzy control algorithm, model error compensation system, which constitutes a Fuzzy-Smith control system. It is a Smith predictor to solve the time delay problem in the calcining process of cement rotary kiln, and the large inertia link of the fuzzy controller to control the object. The structure is the same as the general Smith predictive control, and the fuzzy controller is used instead of the PID controller. This control strategy has played the role of fuzzy control, good dynamic performance, strong anti-interference ability and good robustness. It also uses Smith predictor to achieve a control strategy for dynamic compensation of hysteresis. Both of them can overcome the adverse effects brought by the change of the parameters of the controlled object to a certain extent, and also play two roles in the controller and predictor.

A two-dimensional fuzzy controller is used in this paper. The input of fuzzy controller is e of 
kiln temperature error and the change rate of error $\Delta \mathrm{e}$, and the output variable is $\mathrm{u}$. The fuzzy subset of the input variables E, Ec and the output $\mathrm{U}$ are set to $\{\mathrm{NB}, \mathrm{NM}, \mathrm{NS}, \mathrm{Z}, \mathrm{PS}, \mathrm{PM}, \mathrm{PB}\}$. The domains of $E$ and $E c$ all are $\{-6,-5,-4,-3,-2,-1,0,1,2,3,4,5,6\}$, and the domain of $U$ is $\{-7$, $-6,-5,-4,-3,-2,-1,0,1,2,3,4,5,6,7\}$. Its membership functions all choose the triangle function.

The fuzzy control rule table, as shown in Table 1, can be set up by the technical requirements of cement production and the basic principle of fuzzy control.

Table 1 Fuzzy control rule table

\begin{tabular}{|c|c|c|c|c|c|c|c|c|}
\hline & \multirow[b]{2}{*}{$\mathrm{U}$} & \multicolumn{7}{|c|}{$E$} \\
\hline & & NB & NM & NS & $\mathrm{ZO}$ & PS & PM & PB \\
\hline \multirow{7}{*}{$\mathrm{EC}$} & NB & PB & PB & $\mathrm{PM}$ & PM & PS & $\mathrm{ZO}$ & $\mathrm{ZO}$ \\
\hline & $\mathrm{NM}$ & PB & $\mathrm{PB}$ & $\mathrm{PM}$ & PS & PS & $\mathrm{ZO}$ & NS \\
\hline & NS & PM & PM & PM & PS & $\mathrm{ZO}$ & NS & NS \\
\hline & $\mathrm{ZO}$ & PM & PM & PS & $\mathrm{ZO}$ & NS & $\mathrm{NM}$ & NM \\
\hline & PS & PS & PS & $\mathrm{ZO}$ & NS & NS & $\mathrm{NM}$ & $\mathrm{NM}$ \\
\hline & $\mathrm{PM}$ & PS & $\mathrm{ZO}$ & NS & NM & NM & NM & NB \\
\hline & PB & $\mathrm{ZO}$ & $\mathrm{ZO}$ & NM & NM & NM & NB & NB \\
\hline
\end{tabular}

\section{Improved Fuzzy-Smith Controller}

Although the fuzzy controller can overcome the adverse effects caused by the mismatch of the model, it only achieves a certain effect only when the model mismatch is small. With the increase of the model error, the control effect is obviously decreased. Because the original Smith predictor feedback channel signal does not take account of the influence of past or historical deviations on the prediction value, so when the model mismatches, the original Smith control structure is unreasonable. The quality of the Smith predictive compensation control system will deteriorate and even lose its stability. In order to overcome this shortcoming, this paper improves the Smith predictor and introduces an inertial link in the main feedback channel. The inertia link is $1 /\left(t_{f} s+1\right)$. In the new control scheme, taking into account of the influence of the past deviations, the first order inertia link is used for the low pass filtering of the deviation.

As shown in Fig. 2, when there is no error in the Smith model and the object model, TE is zero. There is no difference between the improved scheme and the ordinary Smith predictor. Only when the model mismatches, that is, time constant, time delay and magnification, is there any error in any one of them, TE value is not equal to zero, and inertia links play a role. If the mismatch of the model is caused by the magnification, the introduction of the inertial link cannot improve the control effect, but will decrease the control performance. At this time, only the improvement of fuzzy controller can overcome the adverse effects of magnification. For the model mismatch caused by other parameters, the introduction of inertial link has a great improvement on the performance of the system. Therefore, an adaptive mechanism is introduced to adjust the value of the filtering time constant according to the cause of the mismatch of the model. $T_{f}$ is the inertia time constant.

The basic principle is that the object output value $\mathrm{y}$ is divided by the model output value YM, if the model mismatch is caused only by magnification, then $y / y_{m}$ equals a constant, and its derivative is zero. According to this, the adaptive mechanism is used to adjust the value of $T_{f}$ to zero, which is equivalent to that the inertia link does not work. If $y / y_{m}$ is not equal to a constant, the model mismatch is caused by other parameters other than the magnification, and the inertial link works as usual, so as to improve the control performance. The value of the filtering time constant can make it equal to the model time delay constant $\mathrm{Tm}$. When the value of ${ }^{T} f$ is too small, the 
filtering effect is not obvious, and the performance of the control is not much better than that of the filter without the introduction of the filter. The excessive value of ${ }^{T} f$ can help eliminate the oscillation and make the response curve smooth, but this increases the adjustment time of the system and is not conducive to the fast tracking of the given value by the output of the object.

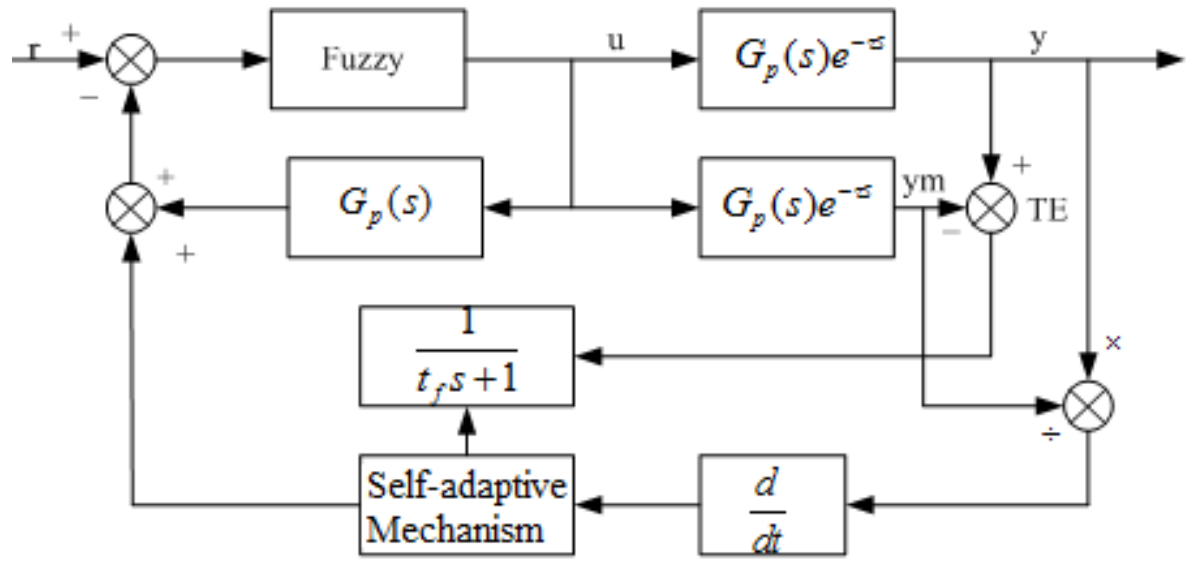

Figure 2. Diagram of the improved Fuzzy-Smith control system

\section{Simulation Study}

The temperature control process of the cement rotary kiln can be approximated by the pure lag and the two order inertia link. The approximate model of the temperature control system of rotary kiln is Eq. 2.

$$
G(s)=\frac{1}{(50 s+1)(8 s+1)} e^{-50 s}
$$

The improved Fuzzy-Smith control scheme is compared with the Fuzzy control scheme, the PID-Smith control scheme and the PID control scheme, and the simulation is carried out in simulink.

The parameters of the PID controller are $\mathrm{Kp}=0.05, \mathrm{Ki}=0.009, \mathrm{Kd}=0.05$. The parameters of the fuzzy controller are $K_{e}=1.2, K_{e c}=0.8, K_{u}=1.2$. The delay time is $\tau=50 \mathrm{~s}$.

Under the same step signal input condition, the four systems are compared with a scope, and the simulation block diagram is shown in Fig. 3.

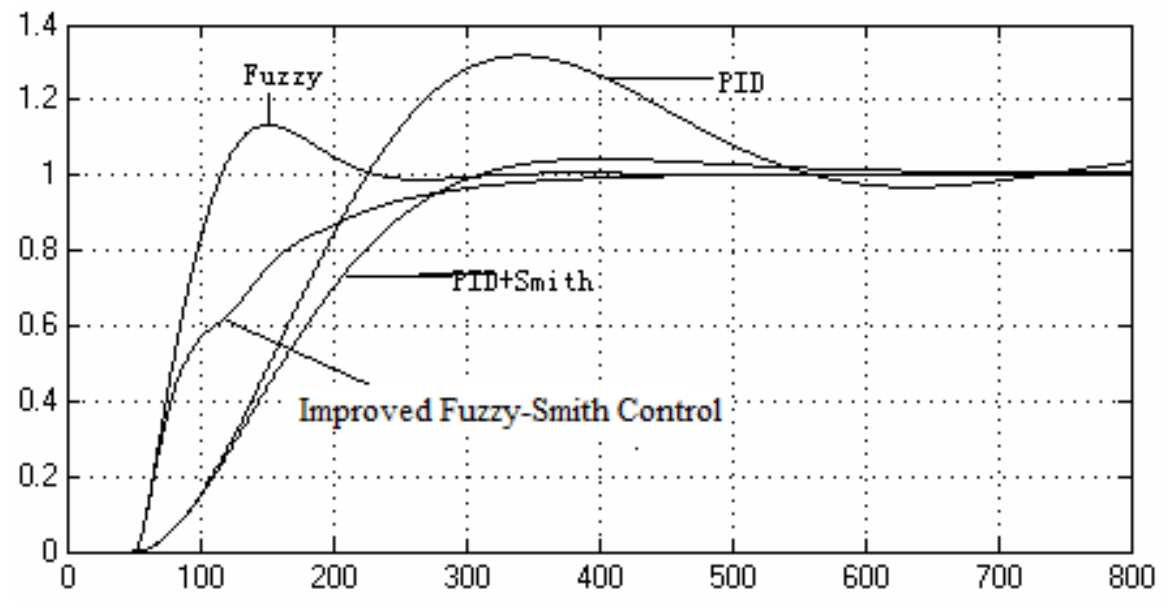

Figure 3. System simulation curve

Compared with the PID-Smith controller, the improved Fuzzy-Smith control decreases the rise time and adjusting time, and greatly reduces the overshoot of the system. Compared with the 
ordinary fuzzy controller, the dynamic and static indexes of the system have been greatly improved when the improved Fuzzy-Smith control is adopted. Therefore, the improved Fuzzy-Smith predictor improved the weaknesses of conventional PID-Smith predictive control, fuzzy control and Fuzzy-Smith control.

\section{References}

[1] B. Bi: Hydromechatronics Engineering, Vol. 42 (2014) No.6, p.37-41.

[2] X.C. Yang: The Control Problem for a Class of Delay System (MS., Zhengzhou University, China 2014 ), p.7-10.(In Chinese)

[3] Y.N. Wang and w. Sun: Intelligent Control Theory and Applications (China Machine Press, China 2017), p.10-18. (In Chinese)

[4] W. Wei: Intelligent Control Technology (China Machine Press, China 2016), p.20. (In Chinese)

[5] T.L. Liu and Y.L. Huang: Computer Simulation, Vol. 27 (2010) No.2, p.170-173. (In Chinese)

[6] M.Y. Ni: Hydromechatronics Engineering, Vol. 41 (2013) No.18, p.153-157.

[7] A.L. Li, F.Yue: 2012 24th Chinese Control and Decision Conference (Taiyuan, China, May 23, 2012). p. 2783-2787.

[8] P.Y. Wang: Computer Simulation, Vol. 28 (2011) No.6, p.247-250.

[9] J.X. Duan: Application Study on Fuzzy PID Controller in Temperature Control System with Large Inertia and Time Delay (MS., Lanzhou University, China 2013 ), p.34. (In Chinese)

[10]L.H. Zhang: Fuzzy PID Temperature Control Algorithms Research Based on the Smith Predictor (MS., Harbin Institute of Technology, China 2012), p.15. (In Chinese) 